\title{
A theoretical study of the reduction mechanism of sulfoxides by thiols.
}

B. Balta, G. Monard, M. F. Ruiz-López, M. Antoine, A. Gand, S. Boschi-

Muller and G. Branlant

\section{Supporting information}

\section{Optimized structures}

Structure 1

$$
\begin{array}{rrr}
2.108459 & -1.081502 & .332709 \\
3.872198 & -1.547804 & .293139 \\
2.047570 & .280218 & 1.028179 \\
-1.790001 & -.968218 & -.382071 \\
-2.479292 & -2.118447 & -.151131 \\
1.905419 & -.777332 & -1.453291 \\
-1.001769 & .766341 & -.983811 \\
-.979308 & 2.139901 & -.292981 \\
-.842618 & 1.820341 & 1.497109 \\
.693573 & 2.829180 & -.529381 \\
.826423 & 2.977110 & -1.600831 \\
.734154 & 3.795070 & -.022481 \\
1.432252 & 2.131739 & -.129081 \\
-.762447 & 2.776701 & 2.016269 \\
-1.761299 & 1.319962 & 1.801419 \\
.032481 & 1.189660 & 1.665079 \\
4.175238 & -1.765804 & 1.316529 \\
4.444639 & -.706914 & -.100491 \\
3.999727 & -2.435534 & -.327601 \\
1.954718 & -1.731862 & -1.979441 \\
.926419 & -.309371 & -1.572461 \\
2.693530 & -.105622 & -1.796941 \\
-4.413981 & -.640905 & -.388911 \\
-4.784942 & -2.121645 & .508479 \\
-3.927831 & -.801146 & 1.321259 \\
-4.052442 & -1.326875 & .375559
\end{array}
$$

Structure 2

$\begin{array}{rr}1 & 6 \\ 2 & 16 \\ 3 & 8 \\ 4 & 6 \\ 5 & 8 \\ 6 & 16 \\ 7 & 6\end{array}$

$$
\begin{array}{r}
3.408723 \\
2.749883 \\
1.366586 \\
2.269639 \\
.160310 \\
-1.170936 \\
-.792656
\end{array}
$$$$
\begin{array}{r}
.761084 \\
-.922448 \\
-.859201 \\
-.996799 \\
.957392 \\
1.665189 \\
3.423272
\end{array}
$$

$-.317394$

$$
\begin{array}{r}
-.230536 \\
-1.025892
\end{array}
$$

1.513464

.071911

$-.342037$

$-.110147$ 


$\begin{array}{rr}8 & 6 \\ 9 & 16 \\ 10 & 6 \\ 11 & 1 \\ 12 & 1 \\ 13 & 1 \\ 14 & 1 \\ 15 & 1 \\ 16 & 1 \\ 17 & 1 \\ 18 & 1 \\ 19 & 1 \\ 20 & 1 \\ 21 & 1 \\ 22 & 1 \\ 23 & 1 \\ 24 & 1 \\ 25 & 1 \\ 26 & 1\end{array}$

$\begin{array}{rrr}-2.277341 & 1.403136 & 1.063210 \\ -3.009450 & -1.859371 & -.415489 \\ -1.578097 & -2.804415 & .284914 \\ 3.179933 & -.903838 & 2.106305 \\ 1.564891 & -.192451 & 1.721387 \\ 1.818563 & -1.973908 & 1.678628 \\ 4.309219 & .789614 & .296719 \\ 3.660783 & .948593 & -1.359740 \\ 2.653472 & 1.459186 & .040680 \\ .740455 & -.080726 & -.637699 \\ -1.710548 & 3.995030 & -.249632 \\ -.381865 & 3.570632 & .888221 \\ -.066215 & 3.696723 & -.873678 \\ -3.138876 & 2.061995 & .944020 \\ -2.585473 & .355775 & .994561 \\ -1.732559 & 1.627662 & 1.980228 \\ -.659098 & -2.557745 & -.249599 \\ -1.429374 & -2.549649 & 1.337533 \\ -1.732044 & -3.884017 & .218621\end{array}$

Structure 3

$\begin{array}{rr}1 & 6 \\ 2 & 16 \\ 3 & 8 \\ 4 & 6 \\ 5 & 8 \\ 6 & 16 \\ 7 & 6 \\ 8 & 6 \\ 9 & 16 \\ 10 & 6 \\ 11 & 1 \\ 12 & 1 \\ 13 & 1 \\ 14 & 1 \\ 15 & 1 \\ 16 & 1 \\ 17 & 1 \\ 18 & 1 \\ 19 & 1 \\ 20 & 1 \\ 21 & 1 \\ 22 & 1 \\ 23 & 1 \\ 24 & 1 \\ 25 & 1 \\ 26 & 1\end{array}$

-1.831165
-1.247938
.396684
-1.515497
1.983496
3.473777
3.717615
3.669370
-3.773506
-3.514390
-4.105637
-2.459074
-3.795268
-1.739246
-2.867720
-1.169571
-.866879
-1.221009
-2.562102
.938255
3.615426
2.957175
4.722547
3.480563
4.692570
2.945616

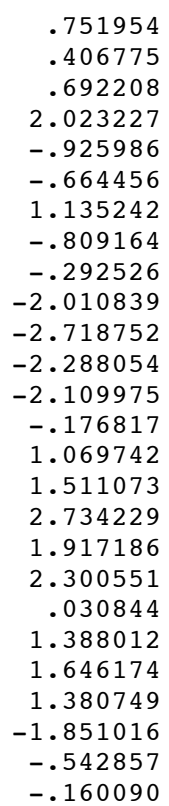

$$
\begin{array}{r}
1.815210 \\
.125701 \\
.529617 \\
-.669899 \\
-.784150 \\
-.492528 \\
-.634553 \\
1.312177 \\
-.627444 \\
-.022438 \\
-.604546 \\
-.121105 \\
1.027968 \\
2.375284 \\
1.772289 \\
2.228583 \\
-.162086 \\
-1.712534 \\
-.597920 \\
.005108 \\
-1.688853 \\
-.042792 \\
-.289694 \\
1.567204 \\
1.578545 \\
1.806167
\end{array}
$$

\section{Structure 4}

$\begin{array}{rr}1 & 6 \\ 2 & 16 \\ 3 & 8 \\ 4 & 6 \\ 5 & 16 \\ 6 & 6 \\ 7 & 8 \\ 8 & 16 \\ 9 & 6 \\ 10 & 6 \\ 11 & 1 \\ 12 & 1 \\ 13 & 1 \\ 14 & 1 \\ 15 & 1 \\ 16 & 1 \\ 17 & 1 \\ 18 & 1 \\ 19 & 1\end{array}$

$$
\begin{array}{rrr}
2.743269 & 1.341009 & .303838 \\
3.837659 & .023249 & -.339112 \\
-.277771 & -1.803711 & -.646752 \\
3.099949 & -1.443641 & .467128 \\
-1.388330 & -2.337102 & .507008 \\
-2.814310 & -2.744652 & -.542512 \\
-.529331 & .851159 & -.674632 \\
-1.807811 & 1.569258 & -.203422 \\
-1.550251 & 3.318428 & -.628582 \\
-1.673051 & 1.716438 & 1.605368 \\
3.128899 & -1.333491 & 1.552318 \\
2.075249 & -1.601311 & .128208 \\
3.713550 & -2.299421 & .182988 \\
2.813439 & 1.393779 & 1.391578 \\
3.102549 & 2.280159 & -.118652 \\
1.709449 & 1.178239 & -.003682 \\
-.382201 & -.819331 & -.703322 \\
-3.236551 & -1.851842 & -1.005142 \\
-2.528730 & -3.466582 & -1.307842
\end{array}
$$




$\begin{array}{ll}27 & 1 \\ 28 & 1 \\ 29 & 1 \\ 30 & 1 \\ 31 & 1 \\ 32 & 1\end{array}$

Structure 6'

$\begin{array}{rr}1 & 6 \\ 2 & 16 \\ 3 & 6 \\ 4 & 16 \\ 5 & 6 \\ 6 & 8 \\ 7 & 8 \\ 8 & 16 \\ 9 & 6 \\ 10 & 6 \\ 11 & 16 \\ 12 & 6 \\ 13 & 1 \\ 14 & 1 \\ 15 & 1 \\ 16 & 1 \\ 17 & 1 \\ 18 & 1 \\ 19 & 1 \\ 20 & 1 \\ 21 & 1 \\ 22 & 1 \\ 23 & 1 \\ 24 & 1 \\ 25 & 1 \\ 26 & 1 \\ 27 & 1 \\ 28 & 1 \\ 29 & 1 \\ 30 & 1 \\ 31 & \end{array}$

Structure 7

$\begin{array}{rr}1 & 6 \\ 2 & 16 \\ 3 & 16 \\ 4 & 6 \\ 5 & 6 \\ 6 & 1 \\ 7 & 1 \\ 8 & 1 \\ 9 & 1 \\ 10 & 1 \\ 11 & 1 \\ 12 & 1 \\ 13 & 1 \\ 14 & 1\end{array}$

Structure 8

$\begin{array}{rr}1 & 16 \\ 2 & 6 \\ 3 & 1 \\ 4 & 1 \\ 5 & 1\end{array}$

2.168102
1.333441
.987230
1.344448
.089258
.603939
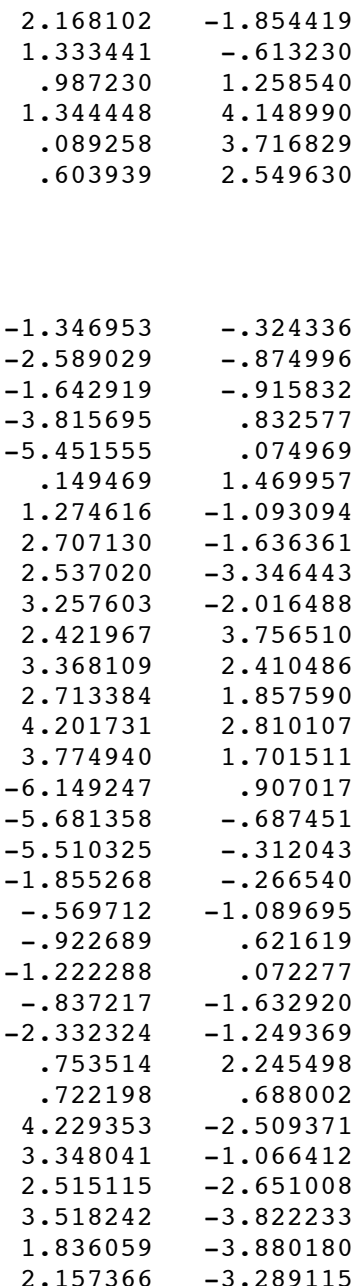

2.157366
$-.324336$

$-.874996$

$-.915832$

.832577

.074969

1.469957

$-1.093094$

$-1.636361$

$-3.346443$

$-2.016488$

3. 756510

2. 410486

1. 857590

2. 810107

1. 701511

.907017

-.687451

$-.312043$

$-.266540$

$-1.089695$

.621619

.072277

$-1.632920$

2. 245498

.688002

$-2.509371$

$-1.066412$

$-2.651008$

$-3.822233$

$-3.880180$

$-3.289115$
$-1.249369$
2.362301

1. 361221

$-1.037189$

1.875961

.722471

1. 953211

1. 406899

.195722

$-1.359350$

$-.046673$

.276230

$-.608704$

-. 145178

$-.276141$

$-.875443$

1. 417321

$-.296696$

.553994

1. 231632

1.134908

$-.171014$

.167154

$-.464219$

1.290655

2. 366740

1. 403247

1.067958

$-1.544092$

$-1.199146$

$-2.131683$

$-.570749$

$-.503092$

1. 371538

1. 941905

1. 901845

$-.872025$

$-.233232$

$-1.894573$
1.621092
.631130
-1.088664
-2.382411
1.548809
-3.311257
-2.334920
-2.328925
1.103505
1.721008
2.591421
2.498254
.958927

1.697586

$$
\begin{array}{r}
-1.391283 \\
.024821 \\
-.094084 \\
.066733 \\
1.438278 \\
-.008494 \\
1.037228 \\
-.751628 \\
-2.288391 \\
-1.364824 \\
-1.310247 \\
1.479488 \\
2.327303 \\
1.305407
\end{array}
$$

$$
\begin{array}{r}
.057963 \\
-.514701 \\
.710781 \\
-.573904 \\
.172688 \\
-.005486 \\
-1.061256 \\
-1.287580 \\
-.274222 \\
1.140888 \\
-.431482 \\
-.360983 \\
-.039682 \\
1.242051
\end{array}
$$

Structure 9

$\begin{array}{rrr}.000000 & .000000 & .715190 \\ .000000 & .000000 & -1.141729 \\ .000000 & 1.020318 & -1.530889 \\ .883621 & -.510159 & -1.530889 \\ -.883621 & -.510159 & -1.530889\end{array}$




$\begin{array}{rrr}.002003 & -.263430 & -.007271 \\ -2.470506 & -.082086 & -.104919 \\ -2.466375 & 1.744039 & .068593 \\ -3.489478 & 2.092856 & -.077358 \\ -2.132451 & 2.048299 & 1.060812 \\ -1.833850 & 2.205571 & -.690881 \\ -.036705 & -1.314618 & 1.492594 \\ -.089838 & -.635109 & 2.340975 \\ -.921303 & -1.943589 & 1.455412 \\ .875084 & -1.903599 & 1.533864 \\ -.890495 & -2.097577 & -1.297279 \\ .039912 & -1.537049 & -1.321192 \\ .141123 & -1.002639 & -2.263509 \\ .897694 & -2.183473 & -1.158387 \\ 2.469830 & -.078844 & .036702 \\ 2.460298 & 1.748972 & -.116819 \\ 1.906340 & 2.208831 & .702400 \\ 3.495678 & 2.088540 & -.066041 \\ 2.037487 & 2.063582 & -1.071252\end{array}$

Structure 10

$\begin{array}{rr}1 & 6 \\ 2 & 16 \\ 3 & 8 \\ 4 & 1 \\ 5 & 1 \\ 6 & 1 \\ 7 & 1 \\ 8 & 8 \\ 9 & 16 \\ 10 & 6 \\ 11 & 1 \\ 12 & 1 \\ 13 & 1 \\ 14 & 6 \\ 15 & 1 \\ 16 & 1 \\ 17 & 1\end{array}$

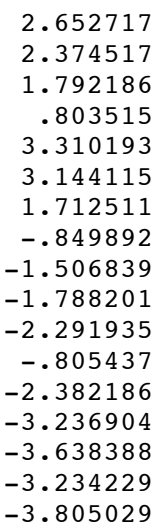

.446608 .327340

$-1.246108$

$-1.193513$

-.355440

1. 407731

.419873

$-1.074340$

.075746

1. 406744

.993720

1.790545

2. 193999

$-.438865$

-. 751173

$-1.272087$

.393635

1. 340521

$-.451065$

$-.598520$

$-.515012$

1. 676764

1. 511894

1.892837

$-.426595$

.358850

$-.850646$

$-1.724883$

$-1.122039$

$-.385519$

.583376

$-.380786$

1. 284386

.999206

Structure 11

$\begin{array}{rr}1 & 16 \\ 2 & 1 \\ 3 & 6 \\ 4 & 1 \\ 5 & 1 \\ 6 & 1\end{array}$

$-.048513$

$-.670717$

$-.846728$

1.288003

$-.048513$

1. 166515

1. 471074

.436789

1. 554019

.436789

1. 554019

.000000

.000000

.000000

.000000

.893695

$-.893695$

Structure 12

$\begin{array}{rr}1 & 16 \\ 2 & 6 \\ 3 & 6 \\ 4 & 1 \\ 5 & 1 \\ 6 & 1 \\ 7 & 1 \\ 8 & 1 \\ 9 & 1\end{array}$

.000000

.000000

.000000
1.400888

$-1.400888$

$\begin{array}{ll}.000000 & -1.400888 \\ .000000 & -2.314634\end{array}$

$-.893442$

$-1.382030$

.893442

.000000

$-1.382030$

2. 314634

.893442

1. 382030

1. 382030

.659649

$-.513454$

$-.513454$

.080956

$-1.138710$

$-1.138710$

.080956

$-1.138710$

$-1.138710$

Structure 13

1

2

$\begin{array}{rrr}1.871873 & .801989 & .385725 \\ .924213 & -.497351 & -.494634\end{array}$




$\begin{array}{rr}3 & 16 \\ 4 & 6 \\ 5 & 1 \\ 6 & 1 \\ 7 & 1 \\ 8 & 1 \\ 9 & 1 \\ 10 & 1\end{array}$

$\begin{array}{rrr}-.924202 & -.497313 & .494652 \\ -1.871896 & .801919 & -.0385783 \\ -1.964115 & .557367 & -1.442475 \\ -2.861679 & .809684 & .074626 \\ -1.408263 & 1.778555 & -.257285 \\ 1.410033 & 1.779112 & .0254499 \\ 2.862754 & .807402 & -.072334 \\ 1.961233 & .559057 & 1.443036\end{array}$

TS1_2

$\begin{array}{rrr}-1.960836 & -2.250000 & -.888112 \\ -2.555122 & -.820172 & .044695 \\ -1.826734 & .473852 & -.588141 \\ -1.778616 & -1.083553 & 1.654733 \\ .614150 & -.953314 & -.159281 \\ 2.074519 & -.503368 & -.336488 \\ 3.039880 & -2.048284 & -.307777 \\ 2.597907 & .154421 & 1.276175 \\ .490172 & 2.563890 & -.588437 \\ -.813495 & 3.484519 & .348407 \\ -2.246170 & -1.970455 & 2.084498 \\ -.707293 & -1.208576 & 1.500809 \\ -2.013629 & -.212019 & 2.263812 \\ -2.425065 & -3.125919 & -.431608 \\ -2.326722 & -2.131720 & -1.906792 \\ -.873764 & -2.279546 & -.836855 \\ -.822958 & .524084 & -.508193 \\ -1.802163 & 3.081246 & .121430 \\ -.651271 & 3.387119 & 1.424884 \\ -.812763 & 4.548054 & .098239 \\ 4.102770 & -1.803818 & -.305358 \\ 2.762762 & -2.628010 & .572853 \\ 2.788299 & -2.595311 & -1.215451 \\ 3.670102 & .354340 & 1.245033 \\ 2.035076 & 1.079091 & 1.399955 \\ 2.361314 & -.571059 & 2.055251\end{array}$

TS2_3

$\begin{array}{rr}1 & 6 \\ 2 & 16 \\ 3 & 6 \\ 4 & 8 \\ 5 & 8 \\ 6 & 16 \\ 7 & 6 \\ 8 & 6 \\ 9 & 6 \\ 10 & 16 \\ 11 & 1 \\ 12 & 1 \\ 13 & 1 \\ 14 & 1 \\ 15 & 1 \\ 16 & 1 \\ 17 & 1 \\ 18 & 1 \\ 19 & 1 \\ 20 & 1 \\ 21 & 1 \\ 22 & 1 \\ 23 & 1 \\ 24 & 1 \\ 25 & 1 \\ 26 & 1\end{array}$

3. 781789

.728023

$-.628134$

3. 049084

$-.915756$

$-.377458$

3. 212663

1. 528855

$-1.021906$

$-.755505$

1. 429183

$-.658843$

1.047360

$-1.172933$

1. 255811

.473878

$-2.163781$

. 315028

$-.137150$

1. 366917

$-1.142794$

$-2.315127$

3. 010241

$-.573553$

$-3.665289$

$-2.569823$

.047889

$-.418913$

1. 677819

1.893334

2.713094

2. 743489

4.833919

3. 690499

3.248856

$-1.041073$

$-.171203$

$-1.955027$

1. 737254

.679505

.958244

1. 464505

.255407

$-1.352065$

$-2.243430$

$-2.209051$

$-2.510487$

$-2.152683$

$-2.634983$

$-3.578198$

3. 300107

3. 589083

$-.468039$

$-3.139998$

3. 118182

1. 717295

.287220

$-.346045$

$-1.688395$

-.026575

$-.026207$

-.355364

1. 134189

-.326299

$-.865435$

.282040

$-1.420869$

1. 100457

$-2.285066$

1.941573

1. 698970 


$\begin{array}{rr}1 & 6 \\ 2 & 16 \\ 3 & 8 \\ 4 & 6 \\ 5 & 16 \\ 6 & 6 \\ 7 & 8 \\ 8 & 16 \\ 9 & 6 \\ 10 & 6 \\ 11 & 1 \\ 12 & 1 \\ 13 & 1 \\ 14 & 1 \\ 15 & 1 \\ 16 & 1 \\ 17 & 1 \\ 18 & 1 \\ 19 & 1 \\ 20 & 1 \\ 21 & 1 \\ 22 & 1 \\ 23 & 1 \\ 24 & 1 \\ 25 & 1 \\ 26 & 1 \\ & \end{array}$

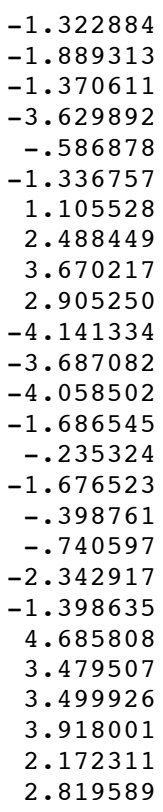

TS2_4

$\begin{array}{rr}1 & 6 \\ 2 & 16 \\ 3 & 6 \\ 4 & 8 \\ 5 & 16 \\ 6 & 6 \\ 7 & 1 \\ 8 & 1 \\ 9 & 1 \\ 10 & 1 \\ 11 & 1 \\ 12 & 1 \\ 13 & 1 \\ 14 & 1 \\ 15 & 1 \\ 16 & 1 \\ 17 & 8 \\ 18 & 16 \\ 19 & 6 \\ 20 & 6 \\ 21 & 1 \\ 22 & 1 \\ 23 & 1 \\ 24 & 1 \\ 25 & 1 \\ 26 & 1\end{array}$

2.298082

2. 716622

2.058453

1. 429610

$-.247963$

$-.144803$

2. 614845

.995984

2.219693

2. 892924

2. 561831

1. 229973

.588520

$-.445735$

$-.793152$

.881437

$-.577788$

$-2.064868$

$-2.752229$

$-2.858986$

$-3.939276$

$-2.585705$

$-2.483436$

$-3.836099$

$-2.302630$

$-1.168707$

-.567306

$-1.903217$

.877252

2. 655620

3.053340

$-2.818466$

$-2.021189$

$-1.604507$

$-2.056716$

$-.373697$

$-1.378958$

.383681

4.087549

2. 394869

2. 925061

$-.926791$

$-.596553$

$-.257784$

$-2.209874$

$-2.071045$

$-2.888284$

$-2.584543$

$-.161745$

.683587

$-2.477498$

$-1.070003$

1. 482610

-. 175750

$-1.213020$

-. 429660

$-.585540$

1. 204920

$-1.006130$

$-1.001550$

$-2.247660$

1. 700160

2.178540

1. 520040

$-.252370$

1. 387240

1. 787130

1. 552560

.155290

.379540

$-1.270160$

.664740

.719200

-.143590

1. 615850

$-1.193000$

$-1.584280$

$-1.943730$

TS5_6

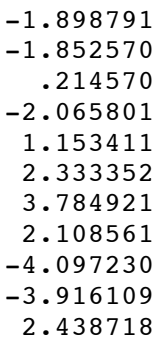

1. 307058

.638489

1.532317

$-1.879940$

$-2.407580$

$-1.403239$

$-1.761420$

$-.230334$

$-1.839884$

2.441480

1.336880

-.174950

$-.254170$

$-1.488110$

$-.987110$

$-.160330$

$-.619680$

1.530800

-. 166050

.691080

$-.503270$ 


$\begin{array}{ll}12 & 6 \\ 13 & 1 \\ 14 & 1 \\ 15 & 1 \\ 16 & 1 \\ 17 & 1 \\ 18 & 1 \\ 19 & 1 \\ 20 & 1 \\ 21 & 1 \\ 22 & 1 \\ 23 & 1 \\ 24 & 1 \\ 25 & 1 \\ 26 & 1 \\ 27 & 1 \\ 28 & 1 \\ 29 & 1 \\ 30 & 1 \\ 31 & 1 \\ 32 & 1\end{array}$

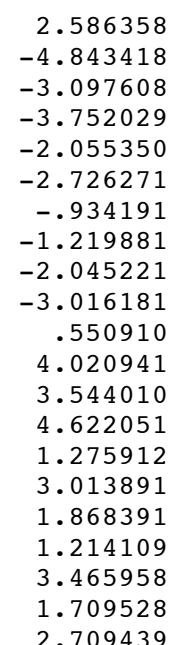

2.709439
2.828891

$-2.392434$

$-2.415983$

$-1.709134$

.625147

2.009087

1.796398

2.208248

.997447

2.047587

$-.222281$

$-1.653218$

$-.341479$

$-1.684418$

$-2.307180$

$-1.944159$

$-.700000$

1.527740

3.454641

3.368240

1.915511

$$
\begin{array}{r}
1.289710 \\
.541700 \\
.256920 \\
1.760250 \\
2.170710 \\
1.274790 \\
1.419610 \\
-1.417930 \\
-2.435710 \\
-1.367150 \\
-.588540 \\
-1.653720 \\
-.529180 \\
.020920 \\
1.972950 \\
2.110200 \\
1.468900 \\
-.409970 \\
1.441760 \\
1.649690 \\
1.873510
\end{array}
$$

1.873510

TS6_10

$\begin{array}{rr}1 & 16 \\ 2 & 6 \\ 3 & 6 \\ 4 & 16 \\ 5 & 6 \\ 6 & 8 \\ 7 & 1 \\ 8 & 1 \\ 9 & 8 \\ 10 & 16 \\ 11 & 6 \\ 12 & 1 \\ 13 & 1 \\ 14 & 1 \\ 15 & 6 \\ 16 & 1 \\ 17 & 1 \\ 18 & 1 \\ 19 & 16 \\ 20 & 6 \\ 21 & 1 \\ 22 & 1 \\ 23 & 1 \\ 24 & 1 \\ 25 & 1 \\ 26 & 1 \\ 27 & 1 \\ 28 & 1 \\ 29 & 1 \\ 30 & 1 \\ 31 & 1 \\ 32 & \end{array}$

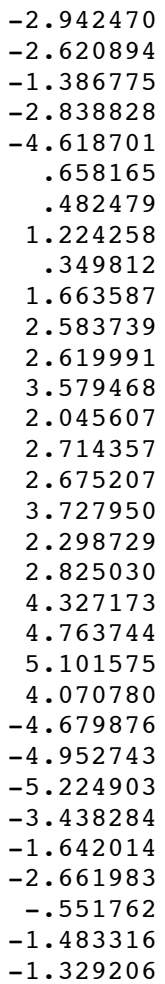

$$
\begin{array}{r}
.204945 \\
1.912323 \\
-.239587 \\
-.960256 \\
-1.379778 \\
-1.201947 \\
-.293250 \\
-1.574860 \\
1.421616 \\
2.225310 \\
1.496484 \\
.406879 \\
1.942001 \\
1.775480 \\
1.629760 \\
.540681 \\
2.005245 \\
2.039947 \\
-2.318064 \\
-1.899431 \\
-.950187 \\
-2.664304 \\
-1.800160 \\
-2.020503 \\
-1.939837 \\
-.492889 \\
2.184011 \\
1.958905 \\
2.524795 \\
-.065798 \\
-1.281797 \\
.398706
\end{array}
$$

.948118

.401620

1.783515

$-.815781$

$-.945515$

$-1.418149$

$-1.121108$

$-.710143$

$-.347630$

$-.240680$

1.148548

1.038124

1.172724

2.054136

$-1.605144$

$-1.619079$

$-1.464055$

$-2.524733$

.806854

$-.190971$

.131615

$-.103811$

$-1.249669$

$-1.826902$

$-.075020$

$-1.115697$

$-.263555$

$-.079583$

1.303443

1.106576

2.079347

2.665125

TS6_13

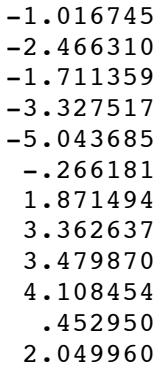

$-1.290617$

1.293119

.229728

$-1.573454$

$-1.844856$

.348618

$-.086477$

.848108

$-.624869$

$-.931125$

$-2.744336$

$-.755720$

3.932423

3.284467

$-1.404754$

.044237

.520827

$-1.025583$

$-.150267$

$-.337449$

$-.469753$

1.315302

$-.264070$

.416555 


$\begin{array}{ll}13 & 1 \\ 14 & 1 \\ 15 & 1 \\ 16 & 1 \\ 17 & 1 \\ 18 & 1 \\ 19 & 1 \\ 20 & 1 \\ 21 & 1 \\ 22 & 1 \\ 23 & 1 \\ 24 & 1 \\ 25 & 1 \\ 26 & 1 \\ 27 & 1 \\ 28 & 1 \\ 29 & 1 \\ 30 & 1 \\ 31 & 1 \\ 32 & 1\end{array}$

$\begin{array}{rrr}2.105909 & 2.202201 & .281743 \\ 2.131160 & 3.484838 & 1.487724 \\ 2.915304 & 3.739761 & -.070139 \\ -5.584066 & .859131 & .448515 \\ -5.481031 & -.793522 & -.180598 \\ -5.093338 & -.451604 & 1.544011 \\ -1.403411 & -1.010517 & 2.270851 \\ -.495112 & -2.245421 & 1.355593 \\ -.394466 & -.521297 & .842842 \\ -1.147229 & -.950377 & -1.668059 \\ -1.076827 & -2.726065 & -1.308563 \\ -2.529799 & -2.049894 & -2.091782 \\ -.177649 & 1.804378 & -.814238 \\ .608710 & .459375 & -.835938 \\ 5.144235 & -1.095395 & 1.283082 \\ 4.073647 & .302990 & 1.567990 \\ 3.522656 & -1.336013 & 2.028601 \\ 4.530370 & -3.036821 & -.478312 \\ 2.950078 & -3.196453 & .368970 \\ 3.011242 & -3.023366 & -1.412421\end{array}$




\section{$\underline{\text { Transition vectors }}$}

\section{TS1_2}

$\begin{array}{crccc}\text { Atom } & \text { AN } & \text { X } & \text { Y } & \text { Z } \\ 1 & 6 & -.08 & -.10 & .04 \\ 2 & 16 & -.02 & -.06 & .01 \\ 3 & 8 & .20 & -.16 & .04 \\ 4 & 6 & -.08 & -.09 & .03 \\ 5 & 8 & -.08 & .13 & -.05 \\ 6 & 16 & -.03 & .05 & -.02 \\ 7 & 6 & -.12 & .00 & .00 \\ 8 & 6 & -.05 & .02 & .00 \\ 9 & 16 & .16 & .16 & -.01 \\ 10 & 6 & -.01 & -.07 & -.02 \\ 11 & 1 & -.12 & -.06 & .04 \\ 12 & 1 & -.08 & -.13 & .06 \\ 13 & 1 & -.05 & -.07 & .02 \\ 14 & 1 & -.14 & -.08 & .02 \\ 15 & 1 & -.03 & -.08 & .03 \\ 16 & 1 & -.09 & -.16 & .08 \\ 17 & 1 & .20 & -.66 & .00 \\ 18 & 1 & .06 & -.25 & -.03 \\ 19 & 1 & .00 & -.04 & -.02 \\ 20 & 1 & -.21 & -.07 & -.02 \\ 21 & 1 & -.11 & -.04 & .01 \\ 22 & 1 & -.15 & .01 & -.01 \\ 23 & 1 & -.13 & .01 & -.01 \\ 24 & 1 & -.04 & -.03 & .02 \\ 25 & 1 & -.01 & .04 & -.02 \\ 26 & 1 & -.09 & .02 & -.01\end{array}$

TS2_3

\begin{tabular}{crccc} 
Atom & \multicolumn{1}{c}{ AN } & X & Y & Z \\
1 & 6 & -.06 & .01 & .00 \\
2 & 16 & -.03 & .00 & .00 \\
3 & 6 & -.08 & .03 & .01 \\
4 & 8 & -.03 & -.04 & -.05 \\
5 & 8 & -.05 & -.11 & -.01 \\
6 & 16 & .00 & .11 & -.05 \\
7 & 6 & -.06 & .05 & -.09 \\
8 & 6 & .21 & .15 & .13 \\
9 & 6 & .03 & -.14 & .02
\end{tabular}




$\begin{array}{rrrrr}10 & 16 & .05 & -.09 & .05 \\ 11 & 1 & -.08 & .06 & .03 \\ 12 & 1 & -.11 & .02 & -.02 \\ 13 & 1 & -.06 & .02 & .00 \\ 14 & 1 & -.07 & .04 & .02 \\ 15 & 1 & -.04 & .00 & -.01 \\ 16 & 1 & -.09 & .01 & -.03 \\ 17 & 1 & -.06 & -.10 & -.05 \\ 18 & 1 & .02 & -.17 & -.01 \\ 19 & 1 & .05 & -.13 & .01 \\ 20 & 1 & -.02 & -.13 & .03 \\ 21 & 1 & .26 & .28 & .08 \\ 22 & 1 & .20 & .04 & .21 \\ 23 & 1 & .28 & .17 & .19 \\ 24 & 1 & .04 & .32 & -.06 \\ 25 & 1 & -.30 & .02 & -.29 \\ 26 & 1 & .03 & -.17 & .04\end{array}$

TS1_4

$\begin{array}{crccc}\text { Atom } & \text { AN } & \text { X } & \text { Y } & \text { Z } \\ 1 & 6 & -.01 & -.01 & .02 \\ 2 & 16 & -.07 & -.19 & -.09 \\ 3 & 8 & .28 & .73 & .20 \\ 4 & 6 & -.02 & .00 & .03 \\ 5 & 16 & -.11 & -.17 & -.05 \\ 6 & 6 & .02 & -.01 & .01 \\ 7 & 8 & .03 & -.01 & -.01 \\ 8 & 16 & .01 & .00 & .00 \\ 9 & 6 & .01 & .00 & .00 \\ 10 & 6 & .02 & .00 & .00 \\ 11 & 1 & -.18 & .10 & .15 \\ 12 & 1 & .04 & .12 & -.04 \\ 13 & 1 & -.02 & -.01 & .01 \\ 14 & 1 & .00 & -.02 & .24 \\ 15 & 1 & -.01 & -.02 & -.01 \\ 16 & 1 & .08 & .11 & -.02 \\ 17 & 1 & .14 & -.03 & .02 \\ 18 & 1 & .00 & .01 & .00 \\ 19 & 1 & -.02 & .10 & .02 \\ 20 & 1 & .14 & -.01 & .17 \\ 21 & 1 & .02 & -.01 & .00 \\ 22 & 1 & .01 & .00 & .00 \\ 23 & 1 & .01 & .00 & .00 \\ 24 & 1 & .02 & -.01 & .02\end{array}$




$\begin{array}{lllll}25 & 1 & .05 & .02 & .01 \\ 26 & 1 & .00 & .00 & .00\end{array}$

TS2 4

$\begin{array}{lllll}\text { Atom } & \mathrm{AN} & \mathrm{X} & \mathrm{Y} & \mathrm{Z}\end{array}$

$\begin{array}{lllll}1 & 6 & .02 & -.03 & .02\end{array}$

$\begin{array}{lllll}2 & 16 & .18 & -.13 & .01\end{array}$

$\begin{array}{lllll}3 & 6 & .02 & -.05 & .00\end{array}$

$\begin{array}{lllll}4 & 8 & -.59 & .61 & -.07\end{array}$

$\begin{array}{lllll}5 & 16 & .15 & -.11 & .02\end{array}$

$\begin{array}{lllll}6 & 6 & .00 & -.02 & -.02\end{array}$

$\begin{array}{lllll}7 & 1 & -.11 & -.16 & -.11\end{array}$

$\begin{array}{llllll}8 & 1 & .01 & .04 & -.03\end{array}$

$\begin{array}{llllll}9 & 1 & .01 & -.03 & .00\end{array}$

$\begin{array}{lllll}10 & 1 & -.09 & -.07 & .19\end{array}$

$\begin{array}{lllll}11 & 1 & .00 & -.01 & .00\end{array}$

$\begin{array}{lllll}12 & 1 & .00 & .06 & .00\end{array}$

$\begin{array}{llllll}13 & 1 & -.11 & -.09 & .02\end{array}$

$\begin{array}{lllll}14 & 1 & -.08 & -.02 & -.18\end{array}$

$\begin{array}{lllll}15 & 1 & .02 & -.02 & -.01\end{array}$

$\begin{array}{lllll}16 & 1 & .01 & .08 & -.02\end{array}$

$\begin{array}{llllll}17 & 8 & -.03 & -.01 & .01\end{array}$

$\begin{array}{lllll}18 & 16 & -.01 & -.01 & .00\end{array}$

$\begin{array}{lllll}19 & 6 & -.01 & -.01 & .00\end{array}$

$\begin{array}{lllll}20 & 6 & -.01 & .00 & .00\end{array}$

$\begin{array}{lllll}21 & 1 & -.02 & -.02 & .00\end{array}$

$\begin{array}{llllll}22 & 1 & -.01 & .00 & .00\end{array}$

$\begin{array}{lllll}23 & 1 & -.01 & .00 & .00\end{array}$

$\begin{array}{lllll}24 & 1 & -.01 & .00 & -.01\end{array}$

$\begin{array}{llllll}25 & 1 & -.03 & -.01 & -.03\end{array}$

$\begin{array}{llllll}26 & 1 & -.01 & -.01 & .00\end{array}$

TS5_6

$\begin{array}{crccc}\text { Atom } & \text { AN } & \text { X } & \text { Y } & \text { Z } \\ 1 & 6 & .00 & .00 & .00 \\ 2 & 16 & .06 & .00 & .00 \\ 3 & 8 & -.14 & -.05 & .02 \\ 4 & 6 & .00 & .00 & .00 \\ 5 & 8 & .00 & -.01 & .00 \\ 6 & 16 & .00 & .00 & .00 \\ 7 & 6 & .00 & .00 & .00 \\ 8 & 6 & .00 & .00 & .00\end{array}$




$\begin{array}{rrrrr}9 & 16 & -.02 & .00 & .00 \\ 10 & 6 & .00 & .00 & .00 \\ 11 & 16 & .02 & .01 & .00 \\ 12 & 6 & .00 & .00 & .00 \\ 13 & 1 & .02 & -.03 & .01 \\ 14 & 1 & .02 & .01 & .01 \\ 15 & 1 & .00 & .00 & .00 \\ 16 & 1 & .00 & .00 & .00 \\ 17 & 1 & -.01 & -.01 & -.01 \\ 18 & 1 & .00 & .00 & .00 \\ 19 & 1 & .00 & .00 & .00 \\ 20 & 1 & .00 & .00 & .00 \\ 21 & 1 & -.01 & -.01 & .00 \\ 22 & 1 & -.14 & -.03 & .01 \\ 23 & 1 & .00 & .00 & .00 \\ 24 & 1 & .01 & .00 & .00 \\ 25 & 1 & .00 & .00 & .00 \\ 26 & 1 & .00 & .00 & .00 \\ 27 & 1 & .00 & .01 & .00 \\ 28 & 1 & -.02 & .00 & -.01 \\ 29 & 1 & .71 & .65 & -.09 \\ 30 & 1 & .00 & .00 & -.06 \\ 31 & 1 & .00 & .00 & -.02 \\ 32 & 1 & .01 & -.01 & -.02\end{array}$

TS6_10

\begin{tabular}{crrrc} 
Atom & \multicolumn{1}{c}{ AN } & \multicolumn{1}{c}{$\mathrm{X}$} & $\mathrm{Y}$ & $\mathrm{Z}$ \\
1 & 16 & -.06 & .08 & .01 \\
2 & 6 & -.09 & .08 & -.02 \\
3 & 6 & -.10 & .17 & .12 \\
4 & 16 & .10 & .05 & .04 \\
5 & 6 & .14 & -.13 & .00 \\
6 & 8 & .21 & .05 & -.17 \\
7 & 1 & .03 & -.03 & -.04 \\
8 & 1 & .11 & -.01 & -.12 \\
9 & 8 & -.07 & -.04 & .06 \\
10 & 16 & -.06 & -.05 & .02 \\
11 & 6 & .01 & -.11 & -.05 \\
12 & 1 & -.01 & -.11 & -.09 \\
13 & 1 & .01 & -.12 & -.08 \\
14 & 1 & .06 & -.14 & -.01 \\
15 & 6 & -.13 & .01 & -.06 \\
16 & 1 & -.22 & .01 & -.16 \\
17 & 1 & -.10 & -.07 & -.05
\end{tabular}




$\begin{array}{rrrrr}18 & 1 & -.13 & .12 & -.01 \\ 19 & 16 & .00 & -.08 & -.04 \\ 20 & 6 & .04 & -.01 & .06 \\ 21 & 1 & .02 & -.02 & .13 \\ 22 & 1 & .05 & -.01 & .04 \\ 23 & 1 & .09 & .06 & .05 \\ 24 & 1 & .23 & -.15 & .01 \\ 25 & 1 & .17 & -.14 & .00 \\ 26 & 1 & .06 & -.19 & -.04 \\ 27 & 1 & -.07 & .04 & -.05 \\ 28 & 1 & -.07 & .08 & .01 \\ 29 & 1 & -.13 & .10 & -.04 \\ 30 & 1 & -.05 & .31 & .21 \\ 31 & 1 & -.02 & .14 & .05 \\ 32 & 1 & -.24 & .12 & .17\end{array}$

TS6_13

$\begin{array}{crccc}\text { Atom } & \text { AN } & \mathrm{X} & \mathrm{Y} & \mathrm{Z} \\ 1 & 6 & .02 & -.23 & -.08 \\ 2 & 16 & -.04 & -.02 & -.06 \\ 3 & 6 & -.11 & -.02 & -.09 \\ 4 & 16 & .14 & .07 & .06 \\ 5 & 6 & .11 & .19 & .07 \\ 6 & 8 & .00 & -.08 & -.08 \\ 7 & 8 & .03 & .06 & .06 \\ 8 & 16 & .02 & .02 & .03 \\ 9 & 6 & -.03 & .01 & .08 \\ 10 & 6 & .06 & .05 & .01 \\ 11 & 16 & -.11 & -.05 & .00 \\ 12 & 6 & -.08 & -.01 & -.03 \\ 13 & 1 & -.09 & -.02 & .04 \\ 14 & 1 & -.03 & .06 & -.05 \\ 15 & 1 & -.10 & -.05 & -.10 \\ 16 & 1 & .19 & .25 & .14 \\ 17 & 1 & .03 & .28 & .04 \\ 18 & 1 & .10 & .14 & .05 \\ 19 & 1 & .09 & -.14 & -.09 \\ 20 & 1 & -.15 & -.32 & -.06 \\ 21 & 1 & .16 & -.35 & -.10 \\ 22 & 1 & -.04 & -.06 & -.07 \\ 23 & 1 & -.19 & -.09 & -.14 \\ 24 & 1 & -.15 & .08 & -.07 \\ 25 & 1 & -.05 & -.08 & -.06 \\ 26 & 1 & .00 & -.05 & -.04\end{array}$


$\begin{array}{lllll}27 & 1 & .05 & .02 & .00\end{array}$

$\begin{array}{lllll}28 & 1 & .09 & .05 & -.02\end{array}$

$\begin{array}{lllll}29 & 1 & .05 & .08 & .04\end{array}$

$\begin{array}{llllll}30 & 1 & -.04 & -.01 & .06\end{array}$

$\begin{array}{lllll}31 & 1 & -.02 & .05 & .10\end{array}$

$\begin{array}{llllll}32 & 1 & -.06 & .00 & .10\end{array}$ 